\title{
Imprudence et impudence
}

Le dispositif ironique dans Les Provinciales

\section{Laurent Thirouin}

\section{(2) OpenEdition Journals}

Édition électronique

URL : http://journals.openedition.org/ccibp/575

DOI : $10.4000 /$ ccibp. 575

ISSN : 2493-7460

Éditeur

Centre international Blaise Pascal

\section{Édition imprimée}

Date de publication : 15 janvier 1996

Pagination : 31-42

ISSN : 0249-6674

Référence électronique

Laurent Thirouin, «Imprudence et impudence », Courrier du Centre international Blaise Pascal [En ligne], 18 | 1996, mis en ligne le 07 janvier 2016, consulté le 02 mai 2019. URL : http:// journals.openedition.org/ccibp/575; DOI : 10.4000/ccibp.575

Ce document a été généré automatiquement le 2 mai 2019.

Centre international Blaise Pascal 


\title{
Imprudence et impudence
}

\author{
Le dispositif ironique dans Les Provinciales
}

\section{Laurent Thirouin}

1 L'art polémique de Pascal dans Les Provinciales se caractérise, selon Jean Mesnard ${ }^{1}$, par deux moyens rhétoriques : «l'expression directe d'une conviction forte», et l'ironie, «à la manière complète qui est celle de Platon ». Qu'est-ce que cette ironie complète? (je parlerais volontiers ici d'une ironie authentique, pour la distinguer de toutes les formes dégradées et dérivées, les plus communes, que l'on gratifie habituellement du nom d'ironie). J. Mesnard précise sa définition, en ébauchant les effets de cette ironie : « Elle a une fonction cathartique; elle guérit de l'aveuglement; elle rend évident ce qui était caché. » C'est donner là à l'ironie une mission d'une singulière ampleur.

2 Goûté de la critique littéraire, le terme d'ironie introduit généralement une grande confusion dans les débats. Convenons simplement, dans un premier temps, que la même notion évoque deux univers rhétoriques bien dissemblables, et qu'entre l'ironie socratique et l'ironie voltairienne ${ }^{2}$ - pour adopter des épithètes conventionnelles - la similitude des mots masque des pratiques très différentes, voire contradictoires.

3 En proposant ici une analyse du "dispositif ironique des Provinciales», je voudrais m'inscrire délibérément dans la perspective d'une définition socratique et montrer comment, bien plus qu'une simple figure du discours, l'ironie dans les Provinciales est le fait d'une véritable stratégie rhétorique ; qu'elle correspond à une conception de la vérité ainsi qu'à un idéal de la correction fraternelle - cette ultime dimension (il faut bien le reconnaître) étant plus caractéristique des Pensées que des Provinciales.

\section{Définitions}

4 Nous ne ferons pas l'économie de la traditionnelle mise au point terminologique qu'impose ce genre de débats. Il importe en effet, pour la suite du propos, de s'entendre sur une définition de l'ironie, celle-ci dût-elle rester une simple définition de nom. La tradition rhétorique, à la suite de Cicéron ${ }^{3}$ et surtout de Quintilien ${ }^{4}$, distingue deux aspects de l'ironie, entendant par cette notion - de façon large - toute forme de 


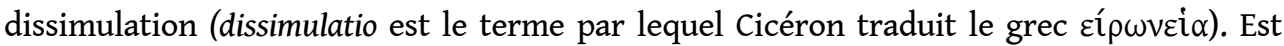
ironique celui qui s'exprime en contradiction avec sa pensée profonde. Quand l'ironie est localisée sur un mot, ou une expression, elle appartient à la famille des tropes, et correspond à notre antiphrase.

L'allégorie où l'on entend le contraire de ce que suggèrent les mots s'appelle ironia : ce qui la fait comprendre, c'est soit le ton de l'énonciation, soit la personne qui s'en sert, soit la nature du sujet ; car, s'il y a désaccord entre l'un de ces éléments et les mots, il est clair que l'orateur veut faire entendre autre chose que ce qu'il dit ${ }^{5}$.

Lorsque, au contraire, c'est toute la démarche de l'orateur qui témoigne une attitude de dissimulation, l'ironie est une figure du discours, et plus précisément, une figure de pensée (figura sententiarum).

Dans la forme figurée de l'ironie, toute l'intention est déguisée [...], la pensée et parfois tout l'aspect de la cause sont en opposition avec le langage et le ton de voix adoptés. Ainsi la vie entière d'un homme peut sembler n'être qu'ironie, comme celle de Socrate (qui était appelé l'ironiste, parce qu'il se présentait comme un ignorant et un admirateur des autres, considérés comme des sages ${ }^{6}$ ).

6 S'inscrivant dans cette tradition, Gérard Ferreyrolles, dans une solide étude sur l'ironie des Provinciales - une des rares, étrangement, que cette vaste question ait jusqu'à présent suscitée ${ }^{7}$ - envisage tour à tour l'ironie « rhétorique » et l'ironie " socratique ", noms qu'il donne respectivement au trope et à la figure. Il n'y aurait d'ailleurs, entre les deux, qu'une différence d'ampleur et non de nature.

7 Cette conception très accueillante de l'ironie présente l'inconvénient de diluer la notion dans des réalités connexes, et surtout de masquer des tensions essentielles. Est-il si certain, comme le soutient Gérard Ferreyrolles, que l'ironie rhétorique (c'est-à-dire finalement l'antiphrase) «ne contredit pas l'ironie socratique, mais s'y intègre ${ }^{8}$ »? Je postulerais plus volontiers pour ma part que ces deux pratiques de l'ironie correspondent à deux objectifs distincts et sont difficilement compatibles. Il me semble en tout cas préférable, méthodologiquement, de ne pas confondre l'ironie elle-même avec ses réalisations les plus habituelles, ou ses effets, que sont l'antiphrase, la raillerie, le persiflage - autant de notions qui ne recouvrent qu'imparfaitement l'ironie comme principe rhétorique.

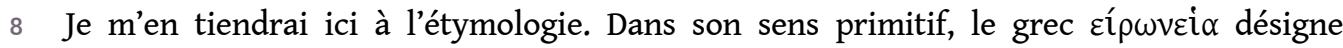
l'action d'interroger en feignant l'ignorance, à la manière de Socrate. Le mot tire son sens de l'adjectif ċi $\rho \omega v$ - dissimulé, qui interroge en feignant l'ignorance - et donne naissance à un verbe $\varepsilon i \rho \omega v \varepsilon \dot{o} \alpha_{\alpha}$ : agir avec une feinte ignorance, faire l'ignorant. Notons d'emblée que cette définition correspond parfaitement au personnage de Montalte dans les premières Provinciales. La quatrième lettre, par exemple, met en scène la perplexité que suscite en lui la "grâce actuelle ».

Je dis à ce bon Père que je lui serais fort obligé s'il voulait m'en instruire et que je ne savais pas seulement ce que ce terme signifiait; je le priai donc de me l'expliquer. (IV, $71^{9}$.)

9 Montalte, rédacteur fictif des Provinciales, questionne et tente de remédier à une ignorance, authentique dans un premier temps, affectée ensuite. La manière de Pascal dans les dix premières lettres est d'ailleurs spontanément rapprochée par $\mathrm{M}^{\mathrm{me}}$ de Sévigné de celle de Platon.

Peut-on avoir un style plus parfait, une raillerie plus fine, plus naturelle, plus délicate, plus digne fille de ces dialogues de Platon qui sont si beaux ${ }^{10}$ ? 
Le caractère essentiel de l'ironie réside donc dans une maligne et feinte connivence avec son interlocuteur, une comédie d'ignorance et de recherche du savoir, dans le but de discréditer le savoir sollicité (le savoir, mais non l'interlocuteur lui-même : la précision est capitale). De son origine socratique, l'ironie garde deux caractères constitutifs : la feinte (faire semblant d'adopter le point de vue de l'autre) et l'intention pédagogique. Un fragment des Pensées, dans une définition imagée, résume ainsi - et j'emprunte encore à J. Mesnard $^{11}$ cette mise en place du problème - l'ensemble des traits du comportement ironique :

On ne s'imagine Platon et Aristote qu'avec de grandes robes de pédants [...] S'ils ont écrit de politique, c'était comme pour régler un hôpital de fous. Et s'ils ont fait semblant d'en parler comme d'une grande chose, c'est qu'ils savaient que les fous à qui ils parlaient pensent être rois et empereurs. Ils entrent dans leurs principes pour modérer leur folie au moins mal qu'il se peut ${ }^{12}$.

11 Platon et Aristote sont des ironistes non tant par leur humeur moqueuse, que par leur feinte adhésion à des principes qui ne sont pas les leurs. Ils affectent un ton qui ne correspond pas à leur humeur. Mais toute cette comédie relève d'une préoccupation médicinale. On sait qu'il est inutile, et même dangereux de contredire un fou; qu'on obtiendra plus de lui en se pliant à ses lubies. L'ironiste ainsi prend notre propre parole pour s'adresser à nous, dans l'intention qu'au bout du compte nous nous déprenions de cette parole ${ }^{13}$.

Dans son sens originel et profond, l'ironie n'est ni raillerie, ni antiphrase, mais une stratégie de dissimulation, qui vise à faire de la victime l'agent essentiel de sa propre défaite. Jean Lafond analyse en ces termes l'ironie de La Rochefoucauld:

L'ironie est un mode d'expression indirect, une feinte qui fait apparaître, en la poussant à sa limite, l'absurdité d'une assertion. Par elle, sont amenés au jour les aspects habituellement occultés d'une pensée qui n'ose pas aller jusqu'au bout d'elle-même et se donner pour ce qu'elle est. S'accommoder au langage de ceux qu'on entend critiquer, c'est se placer sur la position de l'adversaire pour la jouer, et s'en jouer, en la ruinant ainsi de l'intérieur ${ }^{14}$.

13 C'est ainsi que le philosophe Jankélévitch conçoit la position de Socrate, et la fonction de sa méthode d'ironie: désagréger les idées nés d'une ignorance qui ne se sait pas ignorante.

L'ironie, nature composée, s'installe dans l'erreur, non pas pour la comprendre mais pour la perdre ${ }^{15}$.

Les Provinciales certes n'ignorent pas l'antiphrase et l'ironie sommaire, le procédé qui est défini par Fontanier :

L'Ironie consiste à dire par une raillerie, ou plaisante, ou sérieuse, le contraire de ce qu'on pense, ou de ce qu'on veut faire penser ${ }^{16}$.

Mais la plus grande visibilité du procédé masque qu'il s'inscrit dans un dispositif lui-même ironique. C'est quand il est pleinement dissimulé que Montalte est pleinement ironique. Au bout du compte, l'ironie sur laquelle reposent les Provinciales est plus efficace que l'ironie sommaire (celle qui se fonde sur l'antiphrase - l'ironie voltairienne) en cela qu'elle incorpore véritablement la victime dans l'attaque, elle utilise ses principes. Elle est en même temps plus morale, car elle donne le moyen à la victime de s'amender : c'est un art de la répréhension, qui en accordant une partie de la vérité à l'adversaire lui rend la correction acceptable (quoique les jésuites soient trop invétérés dans leur erreur pour en tirer amendement). 
16 Il y a au fondement même de tout procédé ironique une retraite de l'énonciateur, une stratégie citationnelle. En ce sens, les Provinciales qui se construisent sur le jeu des citations, sont un ouvrage structurellement ironique. Cette conception laisse néanmoins subsister une ultime difficulté terminologique : qui est ironique?

17 Sous sa forme authentique, la plus raffinée et la plus diffuse, l'ironie ne correspond véritablement à aucune énonciation représentée. Il n'est donc pas question, dans cette optique, d'isoler un énoncé ironique, comme il serait facile de le faire dans un conte de Voltaire. Soit la phrase du Docteur de Navarre :

La différence qui est entre nous est si subtile, qu'à peine pouvons-nous la marquer nous-mêmes. (I, 45.)

18 On aperçoit sans effort l'argument de Port-Royal sous-jacent : le procès fait à Arnauld est politique et non théologique, à telle enseigne que la divergence doctrinale avec certains de ses adversaires est imperceptible. L'ironie tient à ce que cet argument soit formulé précisément par un de ces pseudo-adversaires d'Arnauld. Mais peut-on dire que le docteur de Navarre s'exprime ironiquement ? En aucune façon. Il exprime une opinion à laquelle il adhère pleinement et dont il tire une conclusion nette : je ne vous explique pas la différence entre les jansénistes et nous, car elle est trop subtile. " Vous auriez trop de difficulté à l'entendre» (I, 45). Montalte, l'interlocuteur, ne profère aucune parole suspecte d'ironie : il est silencieux. Tout au plus peut-on dire qu'il met ironiquement le théologien en position de se ridiculiser. C'est donc la situation elle-même qui est ironique; Pascal, l'auteur des lettres de Montalte, qui agence un dispositif dont l'ironie est que le théologien prononce lui-même les paroles qui le discréditent. Il n'y a pas ici d'énoncé ironique en ce qu'il serait prononcé ironiquement ; il n'y a aucun personnage à la parole ironique, qui « dit le contraire de ce qu' [il] veut faire penser » (pour reprendre la définition de Fontanier).

Questionné sur le duel, le bon père invoque l'autorité de divers casuistes, dont Sanchez, à propos de qui il s'exclame dans son enthousiasme: «Voyez un peu quels gens je vous cite » (VII, 117). Le lecteur comprend évidemment la phrase comme une raillerie, et n'en conçoit aucun respect pour le casuiste. Mais cette expression, qui présente des marques stylistiques certaines de l'ironie (le ton exclamatif, le recours au syntagme familier voyez un peu, opérateur répertorié de l'expression ironique), et qui est de fait une antiphrase, ne peut pas être reçue pour telle dans le contexte narratif : c'est le jésuite qui parle, pour qui Sanchez est bel et bien une autorité imposante. L'ironie est donc ici doublement latente : elle ressort de la réinterprétation ironique d'une expression, à l'encontre de son sens explicite (c'est le cas de toute antiphrase), mais aussi à l'encontre de son intention de signification. Le dispositif bien particulier mis en place par les Provinciales impose donc d'affiner la notion d'ironie.

\section{La rétorsion implicite}

20 Je partirai d'une notion rhétorique stable et nettement définie, celle de "rétorsion ». Ce procédé polémique «consiste à reprendre l'argument de l'adversaire en montrant qu'il s'applique en réalité contre lui ${ }^{17}$. » La rétorsion fait sienne la parole de l'autre et opère un retournement de l'argumentation antagoniste, qu'elle assume explicitement. Si le terme de rétorsion ne figure pas dans le dictionnaire de Furetière, le lexicographe n'ignore pas le procédé et définit le verbe correspondant, «rétorquer", dans un sens strictement technique : 
Se servir contre quelqu'un du même argument qu'il a fait, faire voir qu'il a la même force contre lui.

21 On sait combien Pascal affectionne ce tour de pensée; son œuvre est l'illustration permanente que «toute objection peut être transformée en preuve ${ }^{18}$ ». L'auteur des Pensées maintient une incertitude troublante sur la nature véritable des arguments qu'il manie, dans un univers où la vérité risque toujours d'être établie par cela même qui semblait la ruiner.

Toutes les faiblesses très apparentes sont des forces. (Fr. 268.)

La rétorsion, procédé rhétorique, est élevée dans les Pensées au rang de trait constitutif de la réalité.

Dans les Provinciales, ouvrage polémique, on s'étonnera d'autant moins d'observer des phénomènes de rétorsion caractérisés. Ainsi, les accusations fallacieuses que portent les jésuites contre Montalte amènent celui-ci, dans la onzième lettre, à les leur appliquer à eux-mêmes. Pour justifier le ton des Provinciales, Pascal montre qu'il a parlé avec vérité, discrétion (c'est-à-dire en bannissant tout argument inutilement blessant), en s'abstenant de toute bouffonnerie et en s'attachant à considérer son adversaire avec charité.

Mais si vous voulez, mes Pères, avoir maintenant le plaisir de voir en peu de mots une conduite qui pèche contre chacune de ces règles, et qui porte véritablement le caractère de l'esprit de bouffonnerie, d'envie et de haine, je vous en donnerai des exemples. Et afin qu'ils vous soient plus connus et plus familiers, je les prendrai de vos écrits mêmes. (XI, 182.)

De la même manière, l'accusation de calvinisme se retourne contre ceux qui l'ont d'abord portée :

En vérité, mes Pères, s'il fallait que le soupçon de calvinisme tombât sur eux [les gens de Port-Royal] ou sur vous, je vous trouverais en mauvais termes. (XVI, 264.)

Les conduites tolérées ou prônées par les casuistes jésuites dénotent en effet une absence de respect pour l'eucharistie qui prête bien davantage le flanc à l'accusation de calvinisme. En mettant le débat sur la question du calvinisme, les jésuites suggèrent ainsi de nouvelles charges contre eux-mêmes.

Montalte s'applique donc à lutter contre les jésuites avec leurs propres armes, à adopter leurs principes. S'agit-il de juger Jansénius?

Tout ce que je vous en dirai est qu'il me semble, mon Père, qu'en le jugeant par vos propres règles, il est difficile qu'il ne passe pas pour catholique. (XVIII, 299.)

Faut-il produire des exemples attestant que les papes se trompent parfois?

Pour vous le montrer parfaitement, je vous ferai seulement ressouvenir des exemples que vous-même rapportez dans votre livre. (XVIII, 305.)

Comment l'adversaire pourrait-il résister à des thèses qui sont établies par son autorité, et dont la conclusion est prononcée en son propre nom : "Il est donc constant par vous-même... ", poursuit Pascal dans la dix-huitième Provinciale.

La rétorsion est une modalité de l'argument ad hominem (argument par lequel on confond un adversaire en lui opposant ses propres paroles ou ses propres actes ${ }^{19}$ ), avec la particularité cependant - en tout cas dans les Provinciales - que la rétorsion prend généralement à son compte les principes qu'elle emprunte à son adversaire et qu'elle utilise contre lui. L'emprunt n'est donc pas temporaire et purement rhétorique; il consacre une conformité de pensée réelle entre les adversaires. Mais stricto sensu, la rétorsion n'est pas ironique (même si l'on peut dire communément qu'il y a une ironie du 
sort à se faire retourner contre soi les armes que l'on pensait utiliser contre son adversaire). La rétorsion est un procédé argumentatif, qui utilise les arguments de l'autre - et à cet égard touche à l'ironie. Mais qui, les récupérant, les prenant à son propre compte, les assumant, agit ouvertement et sans feinte.

Les Provinciales deviennent ironiques dès lors que la rétorsion est laissée en suspens. Les propos de l'adversaire s'y prêteraient sans peine. Mais la facilité de cette rétorsion, son évidence sont telles que le lecteur y procédera automatiquement par lui-même. C'est à ce mécanisme que recourt Molière quand, dans le premier acte de Tartuffe, Orgon expose à son frère toutes les marques de vertu données par l'imposteur ${ }^{20}$. Cléante ne discute pas ces éloges, mais la naïveté dont ils témoignent, la personnalité de Tartuffe qu'ils révèlent involontairement, amènent tous les auditeurs à tirer sur le personnage les conclusions inverses de celles qui étaient voulues par Orgon. On peut donc parler d'une rétorsion, mais d'une rétorsion implicite; c'est précisément son caractère implicite qui définit le dispositif comme ironique.

Dans les Provinciales, la rétorsion implicite se manifeste par le détournement d'un argument, qui, produit à des fins apologétiques, est spontanément reçu par l'auditoire comme déconsidérant la cause de celui-là même qui l'utilisait. Un avantage du procédé est que le protagoniste - Montalte - ne perd rien de sa neutralité. C'est comme si les faits parlaient d'eux-mêmes (les discours révèlent leur propre signification). Mais surtout se trouve respecté de la sorte le grand principe rhétorique des Provinciales : les matériaux de l'argumentation sont empruntés aux propres ennemis.

J'ai exposé simplement vos passages sans y faire presque de réflexion. (XI, 176.)

Le rire naît spontanément de la seule lecture des théories jésuites : elles se ridiculisent d'elles-mêmes. Ou pour reprendre les termes que Pascal emprunte à Tertullien :

Que si on y a été excité à rire, c'est parce que les sujets y portaient d'eux-mêmes. ( Ibid.)

R. Duchêne exprime des réserves devant cette justification, faisant remarquer que si les ouvrages des casuistes comportaient réellement un ridicule intrinsèque, ils devraient susciter le rire à la seule lecture, alors qu'ils ne deviennent comiques que dans les Provinciales, à l'issue du travail de choix, de cadrage, de montage effectué par Pascal.

C'est l'auteur qui, par la façon dont il les fait connaître, transforme des traités savants et construits en ouvrages grotesques et comiques. C'est sa mise en œuvre qui fait rire ${ }^{21}$.

Il va de soi que le dispositif ironique des Provinciales est l'effet d'un travail rhétorique, et que le rôle de Pascal ne saurait se borner à celui d'un compilateur. La compilation d'ailleurs, en l'occurrence, est le fait pour une bonne part de son entourage. Il fallait une disposition ingénieuse, pour discréditer des thèses jésuites par leur simple évocation. Comme celui de Socrate, le silence de Montalte n'est pas passivité. La maïeutique socratique fait jaillir la vérité de celui qui ne l'aurait point dite spontanément: il la portait pourtant en lui, mais ne la concevait pas. D'une manière comparable, Pascal révèle une puissance de dérision, qui restait latente dans les traités des casuistes, mais qui ne cesse ensuite de s'y manifester. En cela R. Duchêne n'a pas totalement raison : après la lecture des Provinciales, les ouvrages mêmes des jésuites, sous leur forme originale, ont changé de nature et leur consultation suscite fréquemment le rire. Il n'est pas étonnant que les Provinciales aient fait vendre Escobar!

Je ne sais pas ce qui est arrivé depuis peu, qui fait que tout le monde le cherche. (VI, 100.) 
l'on veut convaincre quelqu'un de son analyse, il ne faut pas omettre de présenter l'analyse adverse et les arguments qui la sous-tendent. L'affirmation, observe Jankélévitch, rend un tout autre son quand elle est passée "par le purgatoire de l'antithèse ${ }^{22} »$. C'est un risque à court terme que l'on prend, celui d'affaiblir sa position en en révélant les faiblesses, ou du moins les points qui peuvent faire l'objet d'attaques. Mais c'est la seule manière d'obtenir chez son interlocuteur une véritable conviction. Une présentation partielle et partiale n'aboutit qu'à une conviction fragile et temporaire. La forme dialogique revêt ainsi un intérêt rhétorique évident : elle garantit la mise en scène de l'autre et de ses arguments. Étant posé ce principe de base, le fonctionnement ironique représente un cas particulier, une situation limite : l'argumentation de l'autre, au lieu d'affronter votre argumentation, de la mettre à l'épreuve, intervient comme un soutien objectif à vos propres thèses. Mettre son adversaire en scène, ce n'est plus prendre un risque, c'est trouver un allié ; sa simple parole renforce la vôtre. On a alors double raison de convoquer l'autre : la raison rhétorique générale qui veut qu'une argumentation soit confrontée à sa contradiction; l'avantage immédiat d'obtenir un renfort inespéré. On gagne sur tous les tableaux. Au lieu d'ébranler, l'adversaire ici « confirme »; le jésuite est le meilleur allié de Montalte.

Dans sa réalisation la plus élémentaire, la rétorsion implicite s'exprime par la technique de l'argument « déplacé ». Un argument en votre faveur a bien plus de poids quand il n'émane pas de votre camp. Cette facile constatation illustrerait une règle fondamentale : on n'est jamais bien défendu par soi-même. Il est aisé pour Pascal, metteur en scène du débat dans les Provinciales, de répartir les arguments dans le respect de ce principe. À qui revient-il, dans la deuxième Provinciale, de dévoiler les intrigues politiques des jésuites ? À un Monsieur N, ami des jésuites, et qui vante leur stratégie dans les termes que pourrait utiliser un ennemi de la Société (II, 53). Il justifie la manière dont la Société " profite » de l'expression de grâce suffisante, mais à l'issue de son discours, le lecteur conclut bien moins à l'habileté qu'à la malignité des stratèges.

Après la censure d'Arnauld, il est de l'intérêt de Port-Royal de faire valoir les conditions contestables dans lesquelles elle a été acquise et le peu de poids qu'on doit lui accorder. Qui se chargera de développer ces arguments dans la troisième lettre ? Ce sont les jésuites eux-mêmes, dont la conduite et les raisons sont ainsi restituées par le docteur neutre :

Mais, après tout, ils ont pensé que c'était toujours beaucoup d'avoir une censure, quoiqu'elle ne soit que d'une partie de la Sorbonne, et non pas de tout le corps; quoiqu'elle soit faite avec peu ou point de liberté, et obtenue par beaucoup de menus moyens qui ne sont pas des plus réguliers ; quoiqu'elle n'explique rien de ce qui pouvait être en dispute quoiqu'elle ne marque point en quoi consiste cette hérésie, et qu'on y parle peu de crainte de se méprendre. (III, 69.)

Ce que pourraient alléguer Arnauld et ses amis contre la censure de la Sorbonne est ainsi mis dans la bouche des jésuites, minimisé certes, mais frappé en même temps du sceau de l'objectivité, que vos ennemis seuls peuvent mettre à votre cause. 
jésuites se font de convaincants porte-parole d'Arnauld, Montalte en revanche est bien moins efficace dans sa tentative symétrique de prendre la défense des jésuites. La cinquième lettre le met en scène, tel un piètre avocat qui amène l'accusation sur la voie la plus dangereuse pour ses clients :

Il ne me resta à dire pour leur défense, sinon que c'étaient les sentiments de quelques particuliers, qu'il n'était pas juste d'imputer au corps. [...] Ce fut sur cela qu'il me découvrit l'esprit de la Société. $(\mathrm{V}, 85$.

Tous ces exemples ont en commun d'opérer un déplacement des arguments, en les confiant à des interprètes inattendus. On verra là le premier stade et le cadre général du dispositif ironique, dont émerge comme réalisation parfaite la figure emblématique des Provinciales qu'est la rétorsion implicite.

42

exposer, dans la septième lettre, les nouvelles maximes concernant le duel, le jésuite souligne l'exploit qu'ont réalisé les casuistes en réconciliant deux notions aussi contradictoires que la piété et le souci mondain de l'honneur. Mais Montalte, qui manifeste un étonnement poli, ne se montre pas suffisamment conscient de la difficulté de l'entreprise.

Elle vous étonne? me dit-il. Je le crois. Elle en étonnerait bien d'autres. Ignorez-vous que d'une part la loi de l'Évangile ordonne de ne point rendre le mal pour le mal, et d'en laisser la vengeance à Dieu? Et que de l'autre les lois du monde défendent de souffrir les injures, sans en tirer raison soi-même, et souvent par la mort de ses ennemis? Avez-vous jamais rien vu qui paraisse plus contraire? Et cependant quand je vous dis que nos Pères ont accordé ces choses, vous me dites simplement que cela vous étonne. (VII, 113.)

Tout ce développement, censé établir la prouesse des casuistes, démontre au premier chef leur mépris de l'Évangile et l'incompatibilité de leurs maximes avec celles de Jésus-Christ. Ce qu'exprime le jésuite est précisément ce que ses adversaires lui opposeraient pour le déconsidérer. Ses arguments pourraient lui être rétorqués. Mais ils ne le sont pas: la rétorsion reste implicite. Aucun porte-parole de Port-Royal ne vient relever combien de tels principes sont condamnables et discréditent toutes les conséquences qui peuvent en être tirées. L'ironie est pleine en ce que le propos se désavoue dans sa propre profération. Le jésuite développe de lui-même et sans intervention les arguments qui réfutent son discours.

Le procédé est efficace; il ne fait que systématiser le principe du déplacement des arguments en confiant à l'accusé lui-même le soin de dresser le réquisitoire. Le mieux informé sur ses turpitudes et le plus soucieux de n'en pas subir la sanction, le jésuite porte à son encontre des accusations de poids. Forgé par Pascal, l'interlocuteur de Montalte se prête évidemment au jeu. Il n'est guère difficile de mettre des paroles maladroites dans la bouche d'un personnage que l'on invente. Un autre exemple de rétorsion implicite ne sera donc pas inutile pour donner la pleine mesure du phénomène. L'ouverture de la cinquième lettre est à cet égard très éloquente.

Voici les premiers traits de la morale des bons Pères jésuites, de ces hommes éminents en doctrine et en sagesse qui sont tous conduits par la sagesse divine, qui est plus assurée que toute la philosophie. Vous pensez peut-être que je raille je le dis sérieusement, ou plutôt ce sont eux-mêmes qui le disent dans le livre intitulé Imago primi saeculi. Je ne fais que copier leurs paroles aussi bien que dans la suite de cet éloge : C'est une société d'hommes ou plutôt d'anges, qui a été prédite par Isaïe en ces paroles : Allez, anges prompts et légers. La prophétie n'en est-elle pas claire ? Ce sont des esprits d'aigles [...] Ils ont changé la face de la Chrétienté. Il le faut croire puisqu'ils le disent. $\left(\mathrm{V}, 84^{23}\right.$.) 
Tout ce premier paragraphe fonctionne comme une affectation d'ironie désamorcée (une pseudo-raillerie), qui se révèle au bout du compte une authentique ironie, dont on avait méconnu la nature et dont les responsables restent inconscients. Montalte laisse croire qu'il se moque des jésuites, par un éloge que son excès même imposerait d'interpréter comme une raillerie. Erreur! L'antiphrase présumée par le lecteur n'en était pas une. «Vous pensez peut-être que je raille je le dis sérieusement, ou plutôt ce sont eux-mêmes qui le disent... » On ne peut soupçonner les jésuites de s'adresser à eux-mêmes des éloges empoisonnés, destinés à être compris comme des blâmes. L'antiphrase laisse la place à une autre forme d'ironie : l'interprétation à charge d'un propos innocent - une rétorsion à proprement parler, mais qui tient sa force ironique de n'être à aucun moment explicitée. Les auteurs de l'Imago primi saeculi font cependant pire que de se ridiculiser par une maladroite auto-glorification; ils révèlent sans le vouloir ce qui est pour Pascal une des perversités essentielles de leur doctrine : leur refus de la tradition de l'Église, la création pour ainsi dire d'une nouvelle forme de religion, qui les apparente à des hérétiques. En proclamant qu'ils ont « changé la face de la Chrétienté », ils donnent crédit à un grief majeur de leurs adversaires - ce dont Montalte prend acte sans autre commentaire «Il le faut croire puisqu'ils le disent. » Le double sens n'est pas élucidé ; l'expression critique est laissée entièrement sous la responsabilité de ses auteurs, afin qu'ils ne soient soulagés en rien des dommages qu'elle leur cause; afin que la manifestation - aussi légère soit-elle - d'une volonté polémique, ne vienne pas jeter la confusion dans un aveu aussi terrible.

Grâce à cet extrait, on percevra d'autant mieux la distance qui sépare l'antiphrase de l'ironie pleine, qu'à côté des rétorsions implicites analysées jusqu'ici, subsiste une remarque de Montalte où l'ironie prend la forme élémentaire de l'antiphrase. Les jésuites interprètent comme une prophétie à leur gloire des paroles d'Isaïe, vagues et que rien ne permet de leur rapporter. "La prophétie n'en est-elle pas claire?» s'exclame malicieusement Montalte, pour souligner par là le délire interprétatif des Pères et leur naïve prétention. La remarque n'a qu'un seul sens, évident quoique contraire à la lettre : l'antiphrase est le moyen éprouvé de la raillerie.

Les propos que tient à Montalte le jésuite des lettres $\mathrm{V}$ à $\mathrm{X}$ donnent un exemple ininterrompu de rétorsion implicite. On relèverait inutilement ce qui apparait à la simple lecture : chacune des paroles du bon Père est désastreuse pour la cause de la Compagnie. Contentons-nous de quelques formules tirées de la lettre $\mathrm{V}$, où le jésuite, qui admet implicitement le ridicule de ses propos («Il n'en faut pas rire », V, 92), nous présente tour à tour ses confrères comme des personnes remarquables par leur ingéniosité (sur la question du jeûne, «ils ont pensé à tout », V, 89), ne se tenant à aucune règle morale ou spirituelle («nous répondons ce qui nous plaît », $\mathrm{V}, 93$ ), et ne parvenant même pas à s'entendre sur leurs propres fantaisies («Ils ne s'accordent presque jamais », V, 91). Le portrait à charge des jésuites se brosse de lui-même, sans presque que Montalte y prête la main. À la limite, la simple nomination des jésuites suffirait à les déconsidérer, sans même qu'ils s'expriment : ainsi peut s'interpréter la fameuse liste de noms qui conclut la cinquième lettre et que Montalte écoute en feignant l'effroi - cas extrême (mais d'une prodigieuse efficacité) de la rétorsion implicite.

Un principe rhétorique essentiel des Provinciales est ainsi d'utiliser comme matériel polémique celui qui est fourni par l'adversaire (qu'on fasse semblant de l'y trouver - comme dans les rencontres fictives des dix premières lettres - ou qu'on l'y trouve 
effectivement et qu'on le présente de façon plus classique sous la forme de citations glanées au fil des ouvrages).

\section{Persiflage}

Au bon Père qui expose les moyens de faciliter «l'usage des choses saintes ", Montalte manifeste sa surprise :

Vraiment, lui dis-je, je ne le croirais jamais, si un autre me le disait En effet, dit-il, cela a quelque besoin de l'autorité de ces grands hommes. (IX, 154.)

Le jésuite reconnaît que les thèses qu'il défend sont si surprenantes, qu'on ne pourrait soi-même, par son propre sens moral, aboutir à de telles maximes. Elles sont surprenantes parce qu'elles sont incroyables, et elles sont incroyables parce qu'elles contredisent toute saine morale. L'hommage que le jésuite rend à ses maîtres signifie le caractère factice et abominable de leur enseignement. Cela rejoint l'exclamation de triomphe de la cinquième lettre : « Eh bien! l'eussiez-vous cru ?» $(\mathrm{V}, 90$.

Notons cependant que c'est Montalte qui amène son interlocuteur à prononcer cette condamnation inconsciente de son camp. Si le jésuite discrédite ainsi ses maitres, c'est entrainé par une réflexion maligne et équivoque : «je ne le croirais jamais, si un autre me le disait "-formule railleuse, puisqu'elle affecte de considérer qu'un jésuite est une autorité considérable, alors qu'elle laisse comprendre le contraire. L'ironie de Montalte est ici moins innocente que nos définitions premières le laisseraient supposer et nous pouvons gager que de lui-même, le bon Père n'aurait point prononcé des paroles si dommageables à sa cause. Cet art malin de rendre l'autre artisan de son propre ridicule reçoit un nom au siècle suivant, celui de persiflage. Avant de devenir un simple synonyme de la raillerie, le "persiflage » a désigné en effet un comportement bien précis. Selon le Dictionnaire de l'Académie de 1762, persifler, c'est « rendre quelqu'un instrument et victime de la plaisanterie par les choses qu'on lui fait dire ingénument. » Quelques années plus tôt, dans son Manuel Lexique, l'abbé Prévost tenait le terme de persiflage pour un

terme nouveau, qui s'est accrédité tout d'un coup à Paris. Je l'ai défini, dans un autre endroit, l'Art, ou l'action de railler agréablement un sot, par des raisonnements et des figures qu'il n'entend pas, ou qu'il prend dans un autre sens ainsi, persifler quelqu'un, c'est le railler, sans qu'il s'en aperçoive. Cependant il semble que sous ce mot on comprend aussi tout badinage d'idées et d'expressions, qui laisse du doute ou de l'embarras sur leur véritable sens ${ }^{24}$.

Et Rousseau, dans un passage très révélateur de l'Émile, après avoir pris la défense des accents ("L'accent est l'âme du discours, il lui donne le sentiment et la vérité ») met en rapport leur proscription en France dans le beau langage avec une forme particulière de raillerie.

C'est de l'usage de tout dire sur le même ton qu'est venu celui de persifler les gens sans qu'ils le sentent ${ }^{25}$.

Le persiflage - on le voit clairement dans l'exemple de Rousseau - se définit par l'inconscience de la victime, ridiculisée à son insu, mais non sans machination ${ }^{26}$.

Le persiflage est ainsi une espèce de l'ironie, conçue au sens large. Celle-ci - qui voit l'adversaire susciter spontanément sa propre déroute - a une dimension quasi miraculeuse (on en précisera bientôt la nature), tandis que celui-là est concerté. Montalte sait trouver les mots qui amèneront son interlocuteur à se trahir. " $O$ que cela est divertissant ! » s'exclame-t-il, après avoir entendu les premières maximes sur le jeûne. Et 
le jésuite de renchérir, conforté par le contentement de son auditoire : «On ne s'en peut tirer [...] je passe les jours et les nuits à le lire ; je ne fais autre chose » $(\mathrm{V}, 90)$. Autrement dit: les maximes des casuistes ont moins pour fonction d'éclairer sur la moralité des comportements, que de procurer une lecture captivante et drôle.

Les Provinciales oscillent de la sorte entre ironie et persiflage plus graves, plus profondes peut-être quand elles respectent un cadre strictement ironique, elles gagnent en drôlerie quand elles mettent en scène une victime manipulée. La première partie de l'ouvrage, celle où la situation se prête le mieux au persiflage, a toujours passé pour la plus divertissante. "Il y a plus d'une nuance, remarque Jankélévitch, entre laisser parler et faire parler ! entre laisser l'adversaire s'enferrer et le «faire marcher $»^{27}$ ! J Jankélévitch, malheureusement, ne précise pas quelles sont ces nuances et quelle est leur signification. Mais les dix-huit Provinciales offrent à cette intuition critique le champ d'application le plus approprié.

\section{La double interlocution}

L'ironie, figure impalpable et d'autant plus raffinée qu'elle est plus discrète, court un risque structurel : celui de n'être pas reconnue. La meilleure ligne de défense d'ailleurs face à un énoncé ironique n'est-elle pas d'affecter l'incompétence rhétorique, en recevant dans son sens apparent ce qui était censé porter une signification seconde? Une ironie méconnue est désamorcée. Le persiflage du XVIII ${ }^{\mathrm{e}}$ siècle exige la présence d'un public de non dupes. Dans ces conditions, le système de double interlocution, développé par les Provinciales, joue comme correctif du dispositif ironique et garantie de son bon fonctionnement rhétorique.

L'ironie tient à ce que l'apologie des jésuites par eux-mêmes équivaut à un discours d'auto-condamnation. Pour préserver l'efficace du dispositif, il ne faut ni expliciter la portée réelle des propos jésuitiques, ni s'affronter au jésuite, sous peine de passer d'une logique de l'ironie à celle du débat. Cette exigence rhétorique correspond d'ailleurs aux nécessités narratives: Montalte doit se taire pour ne pas alerter le jésuite sur le véritable effet de ses paroles et continuer à tirer de lui des preuves accusatrices.

Je souffre toujours les discours [du bon Père] quoique avec bien de la peine. Mais je suis obligé à me contraindre car il ne les continuerait pas s'il s'apercevait que j'en fusse si choqué ; et ainsi je ne pourrais m'acquitter de la parole que je vous ai donnée de vous faire savoir leur morale. Je vous assure que vous devez compter pour quelque chose la violence que je me fais. Il est bien pénible de voir renverser toute la morale chrétienne par des égarements si étranges, sans oser y contredire ouvertement. Mais après avoir tant enduré pour votre satisfaction, je pense qu'à la fin j'éclaterai pour la mienne, quand il n'aura plus rien à me dire. Cependant je me retiendrai autant qu'il sera possible car plus je me tais, plus il me dit de choses. (VIII, 127.)

Il naît entre le jésuite et Montalte une certaine relation de complicité, caractéristique du dispositif ironique, qui se déploie nécessairement dans un échange, dans un dialogue - complicité qui confine même par moments à la sympathie :

Il me fit voir ensuite dans ses auteurs des choses [...] si infâmes que je n'oserais les rapporter, et dont il aurait eu horreur lui-même (car il est bon homme) sans le respect qu'il a pour ses Pères, qui lui fait recevoir avec vénération tout ce qui vient de leur part. Je me taisais cependant, moins par le dessein de l'engager à continuer cette matière, que par la surprise de voir des livres de religieux pleins de décisions si horribles, si injustes et si extravagantes tout ensemble. (VIII, 137.) 
les premières lettres, Montalte tient en fait simultanément deux discours et par cet artifice d'énonciation, il assure malgré tout une manifestation univoque de la vérité. Il joue un double jeu, avec son double interlocuteur: faire parler le jésuite; s'excuser auprès de son correspondant de supporter ces théories.

Cela me fit pitié, mais je ne lui en témoignai rien. $(\mathrm{V}, 96$.

Cela me parut si horrible, que j'eus peine à me retenir mais pour savoir le reste, je le

laissai continuer ainsi... (VII, 119.)

Une mise en scène de la réprobation est opérée, plus efficace qu'un long discours de blâme contre les casuistes. La double énonciation qui caractérise les Provinciales permet de maintenir une situation ironique, tout en filant un propos sous-jacent, ponctué de signaux d'alerte. Coexistent ainsi un discours brut, reproduit sans précaution mais censé se condamner lui-même, et une mise en cause extérieure qui vérifie la bonne perception $\mathrm{du}$ discours brut et assure une part au plaisir de l'indignation.

Mais après avoir tant enduré pour votre satisfaction, je pense qu'à la fin j'éclaterai pour la mienne, quand il n'aura plus rien à me dire. (VIII, 127.)

61 Il est vrai que la neutralité de Montalte s'estompe rapidement au cours des lettres. Le silence du personnage, de méthode pour obtenir des informations, est devenu ruse pour piéger un ennemi. Les Provinciales, roman épistolaire, racontent comment un honnête homme se laisse peu à peu détromper de l'illusion dans laquelle les jésuites l'avaient mis. "Je vous ai cru aussi autrefois », rappelle Montalte au Père Annat, avant de résumer le chemin qu'il a dû parcourir (XVII, 276). Selon R. Duchêne, on peut situer précisément le point de rupture :

C'est la huitième lettre qui est la charnière. C'est là que le personnage « adhère » au parti de Port-Royal. À partir de ce moment-là, et jusqu'à l'explosion finale, le lecteur sait qu'il se retient ${ }^{28}$.

Mais dès la cinquième lettre, l'investigateur endosse son habit de manipulateur.

Je fus ravi de le voir tombé dans ce que je souhaitais, et le lui ayant témoigné, je le priai de m'expliquer... $(\mathrm{V}, 91$.

L'usage de stratagèmes pour obtenir la vérité transforme confusément l'informateur en antagoniste. Au fur et à mesure que la discussion progresse avec le bon Père, Montalte est présenté comme trouvant plus de difficultés à jouer son rôle. Il manque se trahir dans la huitième lettre, par un mauvais dosage de la raillerie, qui alerte sa victime.

Je crois que vous raillez, dit le Père ; cela n'est pas bien. (VIII, 139.)

Et il faut toute la naïveté attribuée au jésuite pour que la comédie puisse se réamorcer et se prolonger encore quelque temps.

L'attitude ironique - nous l'avons signalé d'emblée- comporte une dimension pédagogique fondamentale : elle consiste à prendre les paroles de l'autre pour en faire l'instrument de sa conversion à la vérité. Notons cependant que, restant finalement inconscient du processus ironique auquel il prend part, le jésuite ne peut en retirer aucun bénéfice moral. L'ironie telle qu'elle fonctionne dans les Provinciales n'a pas véritablement cette dimension pédagogique, ou maïeutique, qui lui donnerait sa pleine valeur ${ }^{29}$. En d'autres termes, le jésuite n'est pour ainsi dire jamais considéré comme un interlocuteur à convaincre, mais comme une victime à manipuler pour assurer la conviction d'autres que lui. La différence est notable avec le libertin des Pensées : lui aussi se trouve à maintes reprises ironiquement désavoué par ses propres paroles, mais par cette stratégie de la rétorsion, l'apologiste entend travailler à la conversion et non à la confusion de son contradicteur.

Courrier du Centre international Blaise Pascal, 18 | 1996 
66 À l'indignation contenue vient s'ajouter un deuxième effet, plus classique, de double destinataire : le sous-entendu à l'usage du lecteur. Il s'agit de mots à double entente, ou d'expressions qui ne prennent pas leur sens plein dans leur contexte originel, mais trouvent tout leur sel interprétés par un autre que leur premier destinataire. On est en face d'une raillerie fine, ou d'une ironie au sens plus commun du terme : un énoncé qui signifie implicitement autre chose que ce qu'il semblait vouloir dire.

Voilà un honnête homme, lui dis-je, qu'Escobar. (V, 90.)

67 L' ironie de Montalte prend la forme exacte de l'antiphrase ${ }^{30}$, mais d'une antiphrase que le lecteur est seul à percevoir. Le jésuite persiflé entend pour sa part avec plaisir des paroles moqueuses susceptibles d'une interprétation plus favorable. Montalte n'exprime-t-il pas l'opinion profonde de son interlocuteur, quand il s'écrie :

Que l'Église est heureuse de vous avoir pour défenseurs (VI, 102.)

Mais l'ironie ici a paru outrée à Nicole et aux lecteurs de Port-Royal. Ne supportant pas l'expression aussi brutale - quoique antiphrastique - de thèses honnies, ils ont préféré corriger :

Que le monde est heureux de vous avoir pour maître! (Édition de $1659^{31}$.)

69 Ce phénomène de double destinataire, si caractéristique de la comédie qui se joue jusqu'à la dixième lettre avec le jésuite piégé, ne cesse pas dans la deuxième partie du recueil, quand les lettres changent de ton et de stratégie. Les lettres XI à XVI, qui ne reposent plus sur une mise en scène ironique de la fausse ignorance, conservent cependant - d'une façon explicite quoique différente - la pratique d'une parole qui s'adresse conjointement à deux publics incompatibles. Le destinataire des lettres XI à XVI est à la fois :

- les Révérends Pères jésuites: destinataires officiels, régulièrement rappelés par les apostrophes «mes Pères »

- l'opinion publique: spectatrice de l'échange précédent, dont elle est l'authentique destinataire et l'enjeu véritable.

Jusque dans la syntaxe, le début de la lettre XV montre l'existence de deux destinataires :

Comme la seule chose qui les empêche de rejeter vos médisances est l'estime qu'ils ont de vous, si on leur fait entendre que vous n'avez pas de la calomnie l'idée qu'ils s'imaginent... Ce sera donc, mes Pères, le sujet de cette lettre. (XV, 234.)

71 Montalte s'adresse apparemment aux jésuites, dans un dialogue épistolaire entre un je (ou un on de modestie) et un vous collectif. Mais le public véritable, celui à l'intention duquel cette correspondance a été conçue, transparaît sous la forme d'un eux, pour qui l'auteur ne cherche pas à dissimuler sa sollicitude. Ces deux interlocuteurs, l'apparent et le réel, déterminent chez le polémiste une double stratégie.

Ce que j'en dirai ici [sera] pour montrer votre malice. Je veux seulement vous en faire horreur à vous-mêmes, et faire entendre à tout le monde qu'après cela il n'y a rien dont vous ne soyez capables. (XVI, 251.)

72 C'est la formule de la lettre ouverte: dire à quelqu'un quelque chose qu'il sait déjà, devant des personnes qui l'ignorent, pour montrer par cette audace la véracité de son propos. Pascal ne manque pas d'ailleurs d'en exposer lui-même la théorie.

Et quoique vous sachiez aussi bien que moi ce point de votre morale, je ne laisserai pas de vous le dire, mes Pères, afin que personne n'en puisse douter, en voyant que je m'adresse à vous pour vous le soutenir à vous-mêmes. (XV, 234.)

73 L'ensemble des lettres s'inscrit donc dans un processus de double interlocution, mais selon deux voies rhétoriques distinctes : l'ironie (lettres I à X) ; la force (lettres XI à XVIII). 
La pratique de la lettre ouverte doit se comprendre en effet comme une recherche d'intensité.

Je veux vous le dire à vous-mêmes afin que cela ait plus de force ${ }^{32}$.

Ainsi se vérifie le cadre rhétorique de Jean Mesnard évoqué en introduction.

\section{Sens de l'ironie}

Pour Fontanier, l'ironie est un trope parmi d'autres, dont l'intérêt est comparable à celui de tous les tropes:

Proposer des espèces de mystères, d'énigmes, de problèmes, qui, faciles, et très faciles même à pénétrer, à deviner, à résoudre, le tiennent cependant en éveil, exercent son activité, lui font parcourir, rassembler, rapprocher une foule d'idées, et lui fournissent l'occasion d'un exercice sans travail et sans peine, qui non seulement lui plaît, mais l'enchante, fait ses délices ${ }^{33}$.

Fidèle à sa doctrine, Fontanier interprète les figures de rhétorique dans le cadre restreint de l'elocutio. L'ironie n'y prend place que comme un des nombreux artifices imaginés pour ménager le plaisir du discours. Les Petites Lettres seraient moins plaisantes, si le lecteur accédait à leur sens directement, sans faire le détour des antiphrases et des allusions.

C'est sur un plan plus proprement polémique que l'on perçoit habituellement la fonction de l'ironie dans les Provinciales: un procédé particulièrement efficace pour ridiculiser son adversaire. Patrick Dandrey y voit un rapport avec toute une tradition de l'éloge paradoxal ${ }^{34}$. Ses analyses offrent l'avantage de prendre en compte la dimension socratique des Provinciales, mais elles restreignent le jeu de l'ironie à un de ses aspects seulement (le phénomène de déplacement des arguments ne se laisse pas nécessairement décrire en termes d'éloge) et présentent surtout l'inconvénient d'inscrire dans un horizon exclusivement rhétorique un problème qui, pour Pascal, croise des préoccupations d'une toute autre nature. La notion d'éloge paradoxal (pseudo-encomium) rend compte tout à fait finement de bon nombre d'effets antiphrastiques du texte, mais elle gomme une distinction capitale. Sur un plan philosophique (et non rhétorique), il ne revient pas du tout au même que l'éloge paradoxal soit prononcé par le polémiste ou par ses victimes. Autrement dit, quand Montalte écrit : «Je lui avouai que c'étaient d'habiles gens » (II, 53), et quand le jésuite s'exclame au sujet de ses confrères: "Nous en avons ici de bien subtils " (IV, 83), dans les deux cas l'éloge apparemment fait aux jésuites recouvre une moquerie. Mais cette similitude que souligne le terme de pseudo-encomium masque une différence essentielle entre les deux situations. Ironie et persiflage coexistent dans les Provinciales. Mais le tort subi par les jésuites relève selon les cas de raisons distinctes.

La pratique de l'ironie dans les Provinciales ne ressortit pas simplement à des préoccupations d'ordre rhétorique. Il y a un principe métaphysique derrière la stratégie de la rétorsion implicite ; et la manifestation de ce principe est peut-être le sens ultime du recours à l'ironie.

Dans la deuxième partie des Provinciales, se développe une sorte de discours réflexif sur la pratique ironique des premières lettres, et plus généralement sur la « complaisance » des jésuites, la manière étonnante dont ils fournissent eux-mêmes les preuves dont on a besoin pour les confondre. Pascal feint de s'étonner d'une telle légèreté chez ses adversaires. Au mépris de la prudence la plus élémentaire, les jésuites ne semblent pas se 
préoccuper de dissimuler les motivations réelles de leur comportement, quand bien même celles-ci pourraient leur causer le plus grand tort.

À quoi songez-vous, mes Pères, de témoigner ainsi publiquement que vous ne mesurez la foi et la vertu des hommes que par l'intention qu'on a pour votre Société ? Comment n'avez-vous point appréhendé de vous faire passer vous-mêmes, et par votre propre aveu, pour des imposteurs et des calomniateurs? (XV, 239.)

80 Le conseil railleur, ironique leçon qui n'a pas pour but d'être entendue, est proche de ce que la rhétorique classique désigne sous le nom d'épitrope - à la différence près qu'on ne pousse pas ici le méchant à mal agir, mais qu'on lui indique comment il aurait dû s'y prendre.

81 La surprise de Montalte est d'autant plus forte, que les maladresses des jésuites contredisent rigoureusement l'image initiale donnée par la Compagnie: celle d'une association maléfique et sans scrupule, rendue redoutable surtout par son sens politique. La seule "qualité » que ses ennemis s'accordaient à lui reconnaître était celle de la prudence.

Après ce que vous m'avez dit, j'admire leur prudence et leur politique. (III, 68.)

Et même si, dans la bouche de Montalte, l'éloge n'est pas sans venin, il reconnaît une certaine forme de compétence :

$\mathrm{O}$ mon Père, lui dis-je, voilà qui est bien prudemment ordonné $(\mathrm{V}, 94$.

Essentiellement politiques, les jésuites sont accusés de chercher en permanence à s'accommoder aux personnes et aux circonstances : au lieu de prêcher l'Évangile à temps et à contretemps, ils sont obsédés par la recherche d'une bonne stratégie; ils n'agissent que par calcul. Ils ont érigé leur prudence en système et n'en font même pas mystère, se donnant en quelque sorte, au sein de l'église catholique, comme les champions de la prudence.

Ils couvrent leur prudence humaine et politique du prétexte d'une prudence divine et chrétienne. $(\mathrm{V}, 87$.

Et ce sont les mêmes jésuites, ces héros de la stratégie, qui se révèlent au bout du compte de si mauvais stratèges :

Vous ne savez pas prévoir la suite des choses. (XII, 199)

On conçoit que l'épitrope prenne chez Montalte les accents de l'incrédulité. La prudence supposée masquait en fait une imprudence constitutive.

Je m'étonne donc, non pas de ce que vous leur imposez avec si peu de scrupule des crimes si grands et si faux, mais de ce que vous leur imposez avec si peu de prudence des crimes si peu vraisemblables. Car vous disposez bien des péchés à votre gré, mais pensez-vous disposer de même de la créance des hommes? (XVI, 263-264.)

Dans une telle remarque, il ne faudrait pas négliger la part de la technique polémique; elle consiste ici à déplacer le débat sur des questions mineures, pour établir implicitement que les charges les plus graves sont entendues et hors de discussion: se demander pourquoi les calomnies des jésuites sont invraisemblables, c'est tenir pour acquis que ce sont des calomnies. Mais sous le procédé rhétorique transparait une interrogation grave et essentielle. L'incompétence de l'ennemi est troublante, inquiétante même à la limite, comme tout acte qui semble n'obéir à aucune logique. Ses fautes sont un atout certes, mais aussi une énigme.

87 Les jésuites sont protégés par l'image qu'on a d'eux, le préjugé de moralité qu'on nourrit naturellement pour des religieux. 
[Les gens] se trouvent dans la nécessité ou de croire les crimes incroyables dont vous accusez vos ennemis, ou de vous tenir pour des imposteurs, ce qui leur paraît aussi incroyable. Quoi disent-ils, si ces choses-là n'étaient, des religieux les publieraient-ils, et voudraient-ils renoncer à leur conscience, et se damner par ces calomnies? $(\mathrm{XV}, 233$.

iner cet avantage tactique inestimable que vous possédez, en publiant des théories qui vous condamnent et que vous auriez aussi bien pu laisser secrètes, c'est plus que de l'imprudence, c'est la marque d'un aveuglement. On n'a plus affaire à un simple cas d' imprudence, mais à une étrange manifestation d'impudence.

Ne faut-il pas que vous soyez bien impudents, puisque vous avez fourni vous-mêmes la conviction de votre mensonge par les autres lettres de M. d'Ypres que vous avez imprimées? (XVI, 249.)

Quand ils accusent leurs ennemis des crimes les moins plausibles, et dont il leur est le plus facile de se défendre - comme de ne pas croire en la présence réelle dans l'eucharistie - les jésuites ne semblent même plus craindre que leur malveillance et leurs mensonges soient étalés sur la place publique. Après leur avoir facilement répondu, Montalte ne peut que conclure, une fois encore, à leur impudence.

Cela suffit, mes Pères, pour faire voir clairement qu'il n'y eut peut-être jamais une plus grande impudence que la vôtre. (XVI, 255.)

Le seul comportement que l'on puisse adopter, devant une si troublante et absurde impudence, est de la constater et de la déclarer, en reprenant sans lassitude les paroles du capucin Valérien Magni : mentiris impudentissime.

J'ai arrêté leur impudence, et je l'arrêterai encore par le même moyen. Je déclare donc qu'ils ont menti très impudemment, MENTIRIS IMPUDENTISSIME. (XV, 246.)

La complaisance des jésuites à leur propre défaite suggère à Pascal ce dessein, qu'il exprime très nettement dans la seizième Provinciale, mais qui pourrait résumer la stratégie de toute l'œuvre :

Je veux encore vous faire prononcer cet arrêt à vous-mêmes contre vous-mêmes.

(XVI, 255 35 .)

N'y voyons pas une simple commodité rhétorique, ou la formule d'un surcroît d'efficacité polémique. Dans la possibilité de jeter ce défi ironique, Pascal décèle l'action ultime de la Providence. Les jésuites se détruisent eux-mêmes, miraculeusement, de la même manière que leur maxime de la calomnie perd toute gravité par l'aveu qu'ils en font.

Certainement, mes Pères, vous seriez capables de produire par là beaucoup de maux, si Dieu n'avait permis que vous ayez fourni vous-mêmes les moyens de les empêcher et de rendre toutes vos impostures sans effet. Car il ne faut que publier cette étrange maxime qui les exempte de crime, pour vous ôter toute créance. La calomnie est inutile, si elle n'est jointe à une grande réputation de sincérité : un médisant ne peut réussir s'il n'est en estime d'abhorrer la médisance comme un crime dont il est incapable. Et ainsi, mes Pères, votre propre principe vous trahit. (XVI, 267.)

Rédigeant une défense d'Arnauld, Nicole considérait les divergences et dissentiments à l'œuvre parmi les adversaires du théologien de Port-Royal, et formulait incidemment une règle essentielle :

Il faut remarquer que comme c'est le propre des erreurs de se combattre elles-mêmes aussi bien qu'elles combattent la vérité, ainsi ces Messieurs, quoiqu'unis dans le dessein de flétrir M. Arnauld par une censure, ne s'accordent nullement entre eux du sens auquel ils doivent prendre sa proposition pour la faire condamner ${ }^{36}$.

Courrier du Centre international Blaise Pascal, 18 | 1996 

adversaires d'Arnauld («Voulez-vous donc recommencer nos brouilleries? Ne sommes-nous pas demeurés d'accord de ne point expliquer ce mot de prochain et de le dire de part et d'autre sans dire ce qu'il signifie ? ", I, 48). Mais la raison profonde de cette situation, telle que la présente Nicole, n'est pas conjoncturelle : elle ressortit à une loi métaphysique profonde. «C'est le propre des erreurs de se combattre elles-mêmes aussi bien qu'elles combattent la vérité. » Phrase que l'on doit comprendre dans deux sens : 1 . les erreurs se combattent mutuellement (étant par nature multiples, elles trouvent fatalement d'autres erreurs pour s'opposer à elles) ; 2 . chaque erreur est à elle-même sa propre ennemie.

Vola la raison profonde pour laquelle les jésuites sont en définitive, selon Pascal, inoffensifs; voilà aussi pourquoi ils participent activement à leur propre censure et se prêtent si facilement au jeu de l'ironie. Ce n'est pas un hasard si la calomnie à laquelle ils recourent ne porte pas les mauvais fruits qu'ils en escomptaient, mais c'est l'effet direct d'une loi fondamentale, et tout bonnement providentielle :

Le mal est contraire à soi-même [...] il s'embarrasse et se détruit par sa propre malice. (XVI, 168.)

Des deux sens dont est susceptible la phrase de Nicole, cette formule retient explicitement le second. L'ironie serait particulièrement adaptée à la croisade de Montalte, parce qu'elle est employée contre un adversaire maléfique. Une fois encore, la lecture des Provinciales nous ramène aux considérations de Jankélévitch sur l'ironie.

Si le mal se détruit lui-même, c'est que le mal, en vérité, n'est pas viable ${ }^{37}$.

Que les jésuites soient ainsi les meilleurs auxiliaires de Montalte renforce Pascal dans la conviction que sa cause est juste. Ce n'est somme toute que le corollaire rhétorique de sa croyance religieuse en la victoire inéluctable de la vérité. Toute parole, y compris la plus hostile, renforce à terme la cause de la vérité. Si les jésuites s'attaquent à la conception, qu'ils croient janséniste, de la grâce efficace, rien d'étonnant donc à ce qu'ils obtiennent le résultat inverse et fassent le jeu de leurs adversaires ; à ce que leur propre parole étaye la théorie qu'ils voulaient combattre.

Par cette méprise, voulant ruiner leurs principes, vous les avez vous-mêmes parfaitement établis. De sorte qu'on voit aujourd'hui par une espèce de prodige les défenseurs de la grâce efficace justifiés par les défenseurs de Molina: tant la conduite de Dieu est admirable pour faire concourir toute chose à la gloire de sa vérité.

Que tout le monde apprenne donc par votre propre déclaration que cette vérité de la grâce efficace [...] est si constamment catholique, qu'il n'y a pas un catholique, jusques aux jésuites mêmes qui ne la reconnaisse pour orthodoxe. (XVI, 297-98.)

Cette jubilation d'un homme seul et d'un petit groupe confronté à de puissantes forces organisées, cette conviction d'assister à une espèce de prodige divin, au moment même où la défaite humaine semble se sceller, sont aujourd'hui encore un des traits les plus fascinants et grandioses des Provinciales. Peu importe finalement que les jésuites aient gain de cause, que la violence semble temporairement «opprimer la vérité » (XII, 201). Par leur dispositif ironique, les Provinciales ont pleinement rempli leur rôle. L'ironie, à savoir le bénéfice paradoxal que l'on retire de la parole hostile, est en définitive une prérogative - et donc une manifestation - de Dieu. 


\section{NOTES}

1. Voir: Roger Duchêne, L'imposture littéraire dans les Provinciales de Pascal, $2^{\mathrm{e}}$ éd. revue et augmentée suivie des actes du colloque tenu à Marseille le 10 mars 1984, Presses de l'Université de Provence, 1985. La réflexion de J. Mesnard ici évoquée figure dans la table ronde, p. 387.

2. Je prends l'adjectif "voltairien" comme paradigme usuel de l'ironie, même si l'ironie communément qualifiée de voltairienne ne présente qu'un rapport irrégulier avec l'œuvre réelle de Voltaire. Par "ironie voltairienne», pratique qui coïncide souvent avec l'antiphrase, on entendra les cas où l'interprétation de l'énoncé correspond à l'élucidation des intentions de l'énonciateur.

3. Cicéron, De l'Orateur (II, 269-270).

4. Quintilien, Institution oratoire, livres VIII (6) et IX (1 et 2).

5. Quintilien, institution oratoire, VIII $(6,54)$. Texte établi et traduit par Jean Cousin, « Les Belles Lettres"

6. Quintilien, Institution oratoire, IX $(2,46)$.

7. Gérard Ferreyrolles, "L'ironie dans les Provinciales de Pascal», Cahiers de l'Association internationale des Études Françaises, n 38, mai 1986, pp. 39-50.

8. Op. cit., p. 43.

9. Toutes les références aux Provinciales donnent le numéro de la lettre en chiffres romains, suivi de la page, dans l'édition Le Guern (Folio).

10. Lettre de $\mathrm{M}^{\text {me }}$ de Sévigné à sa fille, du 21 décembre 1689 (O.C., Pléiade III, p. 786).

Pour des rapprochements plus détaillés entre la situation d'énonciation des Provinciales et celle des dialogues platoniciens, voir G. Ferreyrolles, op. cit., p. 44.

11. Jean Mesnard, Les Pensées de Pascal ( $2^{e}$ éd. revue et augmentée) ; SEDES, 1993, p. 286.

12. Fr. 457 (édition Ph. Sellier, classiques Garnier).

13. «L'ironie consiste à adopter le point de vue de l'interlocuteur pour amener celui-ci à le dépasser » (J. Mesnard, ibid., p. 297).

14. Jean Lafond, La Rochefoucauld. Augustinisme et littérature, Klincksieck, 1977, p. 142.

15. Vladimir Jankélévitch, L'Ironie, Flammarion (coll. « Champs »), 1964, p. 117.

16. Pierre Fontanier, Les figures du discours, Flammarion, Paris, 1968, p. 145.

17. Olivier Reboul, Introduction à la rhétorique, P.U.F., 1991, p. 171.

Autre définition « Retourner un argument contre celui qui s'en est servi » (Dupriez).

18. J. Mesnard, op. cit., p. 303.

19. Argument ad hominem qu'il ne faut pas confondre - comme on tend à le faire - avec l'argument ad personam : « attaque contre la personne de l'adversaire et qui vise essentiellement à disqualifier ce dernier » (Ch. Perelman et L. Olbrechts-Tyteca, Traité de l'argumentation, 2 vol. , P.U.F., 1958, p. 150).

20. Tartuffe, acte I, sc. 5 .

21. R. Duchêne, op. cit., p. 221-222.

22. Op. cit., p. 76.

23. Les éléments soulignés le sont par l'auteur, qui signale ainsi les citations des jésuites.

24. Prévost, Manuel Lexique ou Dictionnaire portatif des mots français dont la signification n'est pas familière à tout le monde. $\mathrm{N}^{\text {lle }}$ édition, 1755. Je remercie Denis Reynaud pour cette référence, et pour avoir attiré mon attention sur cet avatar de l'ironie au siècle des Lumières.

25. Rousseau, Émile ou de l'éducation, livre premier. 
26. Épaisseur secrète des mots, il est remarquable que quand Jankélévitch rejette une mauvaise ironie, non philosophique, fondée sur une mise à l'écart d'autrui, c'est le terme de " persiflage » qui vient tout naturellement sous sa plume «L'ironie (celle du moins qui n'est pas dédaigneuse moquerie ou persiflage obscurantiste) l'ironie sollicite l'intellection. » (op. cit. p. 64).

27. Op. cit., p. 101.

28. R. Duchêne, op. cit., p. 375.

29. Là est la limite de l'ironie dans les Provinciales et peut-être l'origine d'un certain malaise chez le lecteur moderne. Nulle réalité durable de cette coopération dans laquelle Jankélévitch voit la marque de la situation ironique : «Tandis que le trompeur laisse le trompé seul et reclus en son erreur, l'ironiste et l'ironisé coopèrent de compagnie à une œuvre commune, à une œuvre d'ironie » (op. cit., p. 65).

30. À quoi s'ajoute la présence d'une expression qu'on peut considérer comme un marqueur d'ironie voilà un $x$ que $\mathrm{N}$.

31. Sur les efforts pour affaiblir l'ironie, dans les corrections de 1659, voir Le Guern, «Les Provinciales ou les excès d'un polémiste abusé ", in Duchêne, op. cit., p. 309-310.

32. Laf. 962 [960] (phrase rayée, au milieu d'une série de notes préparatoires à la quinzième Provinciale, qui ne figurent que dans le Recueil Original ; Ph. Sellier n'a pas inclus cette phrase dans son édition).

33. Pierre Fontanier, op. cit., p. 174.

34. Patrick Dandrey, «Les Provinciales et la tradition de l'éloge paradoxal », in Mélanges offerts à $R$. Duchêne, Tübingen : Günter Narr Verlag, 1992, pp. 467-479.

35. Dans la forme, cette expression évoque la pensée déjà citée : "Je veux vous le dire à vous-mêmes afin que cela ait plus de force. » Le dire à vous-mêmes, vous le faire dire à vous-mêmes : tel est le double dessein rhétorique des Provinciales.

36. Défense de la proposition de M. Arnauld, docteur de Sorbonne, touchant le droit, contre la première lettre de $\mathrm{M}$. Chamillart, docteur de Sorbonne et professeur du roi en théologie. Par un bachelier en théologie de la Faculté de Paris (1656). Texte reproduit partiellement en annexe par R. Duchêne, op. cit., p. 256.

37. Op. cit., p. 97.

INDEX

Mots-clés : Pascal, Provinciales, ironie, rétorsion, persiflage

Keywords : Pascal, Provinciales, irony, retorsion

\section{AUTEUR}

\section{LAURENT THIROUIN}

GRAC, Institut d'Histoire de la Pensée Classique, Université Lumière Lyon 2. 